\title{
MEASURING STARS WITH HIGH ANGULAR RESOLUTION: CURRENT STATUS AND FUTURE PROSPECTS
}

\author{
John Davis \\ Chatterton Astronomy Department \\ University of Sydney
}

\begin{abstract}
The current state of knowledge of angular diameters of stars is reviewed and, based on this review and the requirements for the determination of surface fluxes, effective temperatures, radii and masses, targets of sensitivity, angular resolution and accuracy for future programs of stellar angular diameter measurements are established. Long baseline interferometry is the only technique with the potential to meet all the targets. The necessary improvements in sensitivity, angular resolution and accuracy are promised by the approach adopted in the modern Michelson stellar interferometer under development at the University of Sydney and the prototype instrument, which is currently nearing completion, is briefly described to illustrate how the atmospheric and mechanical problems which have inhibited the development of amplitude interferometry may be overcome using modern technology. This program together with the developments taking place at CERGA lead to the conclusion that the prospects for contributions by high angular resolution measurements to the determination of fundamental stellar quantities during the next decade are excellent.
\end{abstract}

\section{INTRODUCTION}

Since the completion of the program of stellar angular diameter measurements with the Narrabri intensity interferometer, which has been described in the preceding paper (Hanbury Brown 1985), the Chatterton Astronomy Department at the University of Sydney has been engaged in a program to develop a stellar interferometer with increased sensitivity, resolution and accuracy. The primary objective is to develop an instrument capable of contributing to the determination of fundamental stellar quantities, namely emergeant fluxes and effective temperatures, radii and hence luminosities, and masses. Additional studies would include limb-darkening, stellar rotation, extended atmospheres, emission-line stars and Cephieds.

This paper reviews the current status of angular diameter measurements, discusses the targets for future angular diameter determination 193

D. S. Hayes et al. (eds.), Calibration of Fundamental Stellar Quantities, 193-208.

(C) 1985 by the IAU. 
programs and assesses the potential of available techniques. The modern Michelson stellar interferometer under development at the University of sydney is described briefly to illustrate how the targets might be met. Since high angular resolution studies of binary stars are discussed by McAlister (1985) in this symposium the discussion is restricted to single stars. However, it is emphasized that the University of Sydney program is just as concerned with the observation of binary stars (Davis 1983) since for at least the primary components of double-lined spectroscopic binaries it is possible to determine all the gross properties (i.e. mass, radius and luminosity) as demonstrated with the intensity interferometer for $\alpha \operatorname{Vir}$ (Herbison-Evans et al. 1971).

\section{CURRENT KNOWLEDGE OF THE ANGULAR DIAMETERS OF STARS}

Discussion in this section is restricted to direct measurements of angular diameters by interferometry or occultations. A survey of the literature reveals that angular diameters have been measured for some 150 stars with more than half $(\sim 90)$ being determined from lunar occultations. However, many of the published angular diameters are of little interest because of the poor accuracy of their determination. Figure 1 shows the distribution by spectral class of stars whose angular diameters have been determined with published uncertainties better than (a) $\pm 20 \%$ (111 stars), (b) $\pm 10 \%$ (82 stars) and (c) $\pm 5 \%$ (32 stars). In preparing these histograms a weighted mean angular diameter was derived for each star for each observational technique (i.e. for lunar occultations, for speckle interferometry etc.). Where a star had been observed by more than one technique, measurements from the different techniques were not combined and only the most accurate weighted mean angular diameter was taken for the purposes of Figure 1. Combination of measurements from different techniques would only affect the positions of 2 late type stars in Figure 1.

Figure 1 has two distinct ranges. For spectral types earlier than GO the data is dominated by results from the intensity interferometer whereas for spectral types later than GO the majority of the data is from lunar occultations with contributions from the two telescope interferometer at CERGA (I2T), from the amplitude interferometer and from speckle interferometry. All the data for classes G, K and M are for evolved stars.

The data in Figure 1 have been sorted according to the published uncertainties in the angular diameters. Independent checks on the reliability of the data are clearly desirable and ideally the results for individual stars determined by different techniques should be compared. Unfortunately, because of the limitations of the different techniques, there are only a few cases where such comparisons can be made and the relevant data are listed in Table $I$. Mira type stars have been excluded. $\alpha$ Ori has also been omitted because of the apparent variation of angular diameter with wavelength and time (White (1980).

Except for $\rho$ Per and $\alpha$ Her where the speckle and amplitude interferometry results are inconsistent the agreement is generally as good as would be expected. Detailed intercomparison of the results from the 


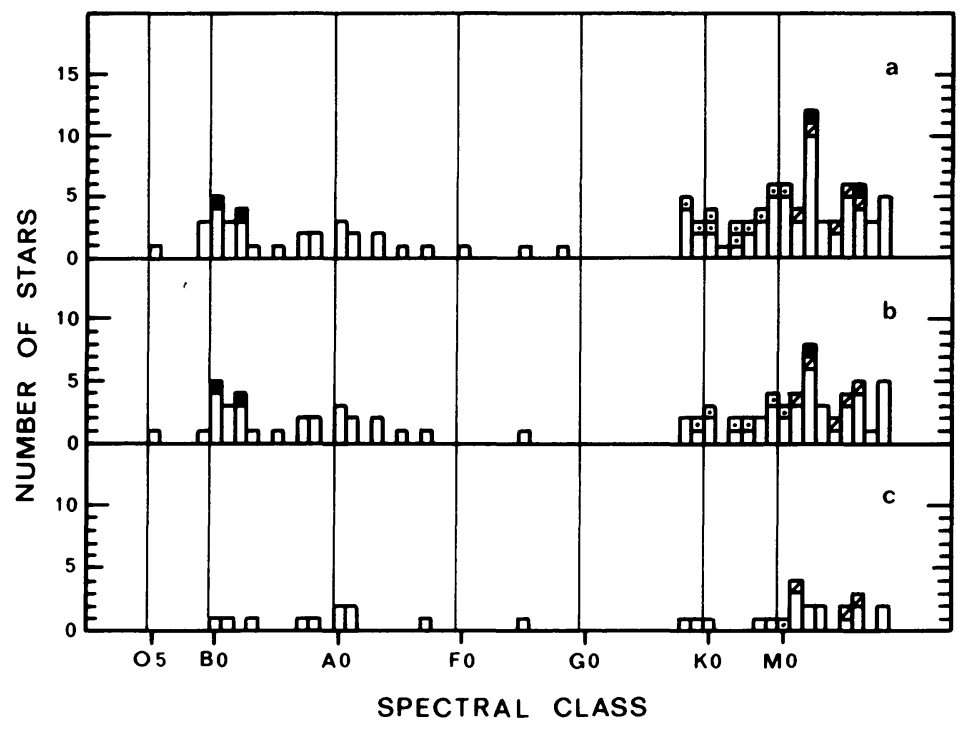

Fig. 1. The number of stars with measured angular diameter as a function of spectral class. The distributions are for angular diameters determined to better than $\pm 20 \%$ (a), $\pm 10 \%$ (b) and $\pm 5 \%$ (c).

For spectral classes O, B, A and F: $\square$ represents measurements by intensity interferometry and $\boldsymbol{D}$ by Jovian occultations. For spectral classes $G, K$ and $M$ : $\square$ represents measurements by lunar occultations, $\square$ by CERGA I2T, $\square$ by speckle interferometry and $\boldsymbol{\square}$ by amplitude interferometry.

different techniques is not warranted because of the small amount of data but Table I serves to emphasize the desirability of obtaining more data, especially for spectral classes earlier than $\mathrm{K}$.

An alternative check on the reliability of data can be obtained by intercomparison of results by the same technique but by different observers with different equipment. This approach is possible for lunar occultations and speckle interferometry although it is limited to late spectral types as shown by the spectral class coverage of the techniques shown in Figure 1. In the case of lunar occultations Ridgway et al. (1980) have compared the error estimates for 66 measurements of 24 stars with the dispersion of measurements for each star. Expressing the deviations from the mean for each star in units of the expected standard deviation, and excluding Mira-type stars, they found excellent agreement between the observed distribution and an appropriately normalized normal distribution for 53 out of 57 observations. The 4 discrepant measurements were for 2 stars and it was concluded that while these results may be the result of real variations it is possible that occasionally ( $\sim 2$ out of 57 ) the occultation technique may be subject to errors which do not reveal themselves in the analysis.

The speckle interferometer results for 3 stars measured by different observers are listed in Table II. $\alpha$ Ori has been omitted for the 


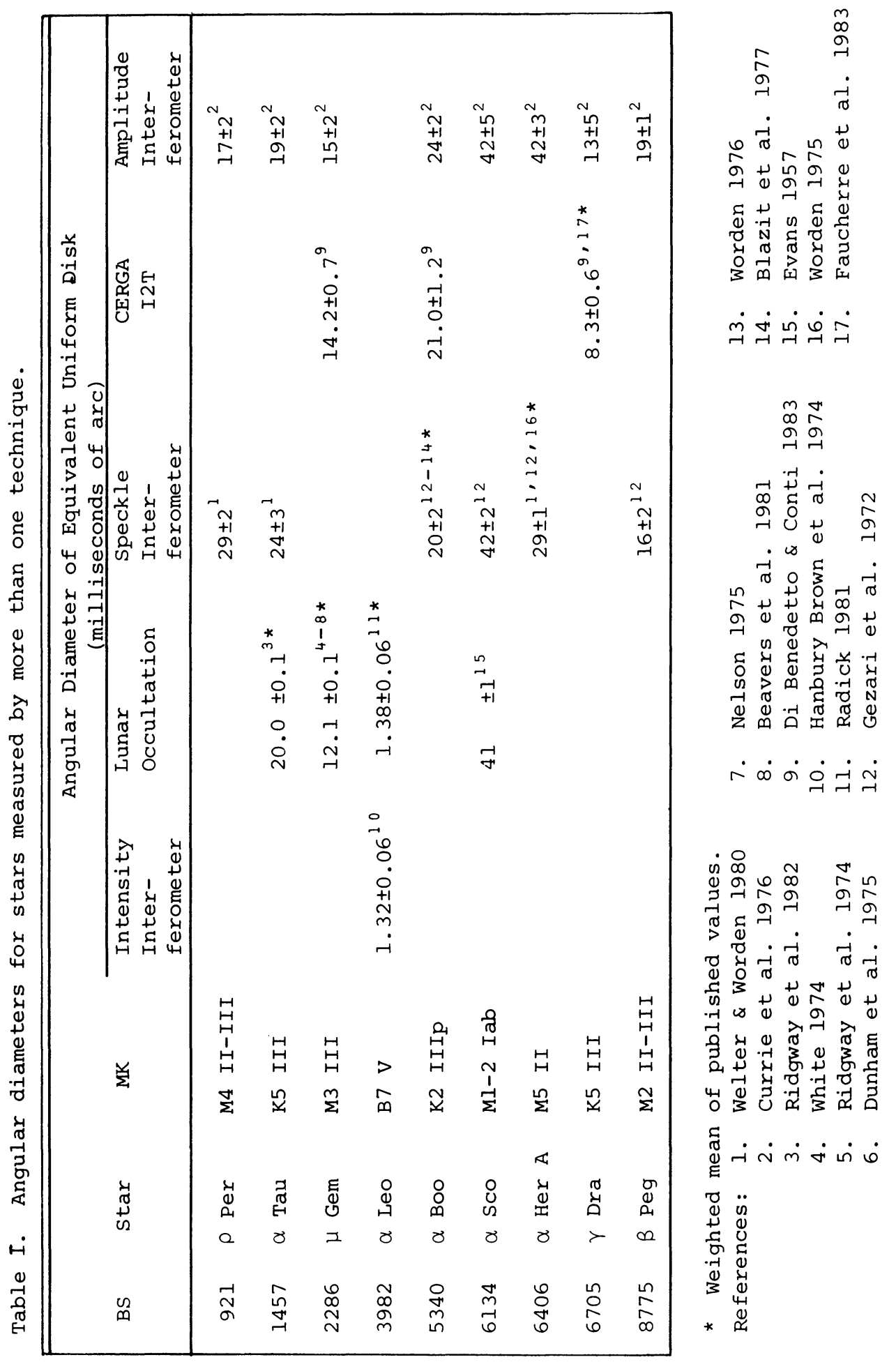


TABLE II. Intercomparison of angular diameters determined by speckle interferometry.

\begin{tabular}{|c|c|c|c|c|c|c|c|c|c|}
\hline \multirow{2}{*}{$\frac{\text { BS }}{911}$} & \multirow{2}{*}{$\frac{\text { Star }}{\alpha \text { Cet }}$} & \multicolumn{2}{|c|}{ MK } & \multicolumn{6}{|c|}{$\begin{array}{l}\text { Angular Diameters } \\
\text { (milliseconds of arc) }\end{array}$} \\
\hline & & M2 & III & $14 \pm 5(400)$ & & $25 \pm 2(550)$ & 2 & & \\
\hline 5340 & $\alpha$ Boo & K2 & IIIP & $\begin{array}{l}18 \pm 4(450) \\
18 \pm 5(500)\end{array}$ & $\begin{array}{l}1 \\
1\end{array}$ & $22 \pm 3(500)$ & 3 & $19 \pm 6(420)$ & 4 \\
\hline 6406 & $\alpha \operatorname{Her} A$ & M5 & II & $\begin{array}{l}29 \pm 1(510) \\
26 \pm 3(520) \\
25 \pm 3(550)\end{array}$ & $\begin{array}{l}2 \\
2 \\
2\end{array}$ & $31 \pm 3(500)$ & 3 & $\begin{array}{l}30 \pm 1(510) \\
28 \pm 3(520)\end{array}$ & $\begin{array}{l}5 \\
5\end{array}$ \\
\hline
\end{tabular}

* The mean wavelength of observation in $\mathrm{nm}$ is given in parenthesis after each result.

References: 1. Blazit et al. 1977

2. Welter \& Worden 1980

3. Gezari et al. 1972

4. Worden 1976

5. Worden 1975

same reason it was omitted from Table I.

Welter and Worden (1980) have commented on the descrepancy between the values for $\alpha$ Cet and noted that the star is close to the limit of their technique. The results for $\alpha$ Boo and $\alpha$ Her $A$ are in reasonable agreement but again there is insufficient data to warrant detailed discussion.

The intercomparison of results between techniques and within techniques, although restricted by the limited amount of data, can be summarized as follows. Although a small number of discordant results exist, the agreement between independent observations of a star is generally in accord with the published error estimates.

Where intercomparison of results is not possible, reliance has to be placed on the internal consistency of data from repeated observations, preferably obtained with modified equipment configurations, and on the efforts taken to eliminate systematic errors from the data. This is particularly true for the results from the intensity interferometer and this is discussed in detail by Hanbury Brown (1985) in the preceding paper.

\section{FUTURE ANGULAR DIAMETER MEASUREMENTS}

Fig. I shows that existina measurements of angular diameters with an accuracy better than $+5 \%$ are spread very thinly across the spectral classes. There is a real paucity of data for spectral classes from late $A$ to late $G$ even if the accuracy is relaxed to $\pm 20 \%$. The situation is 
even worse if only the main sequence is considered since there are no main-sequence stars later than F5 in Figure 1 . However, it should be noted that the Sun (G2 V) and the eclipsing binaries YY Gem (MO.5V) and CM Dra (M4V) have not been included. Angular diameters derived for these eclipsing binaries from trigonometric parallaxes have uncertainties in the $\pm 5-10 \%$ range.

Let us examine the sensitivity, angular resolution and accuracy required to add substantially to our knowledge of stellar surface fluxes and effective temperatures for all spectral types via angular diameter determinations.

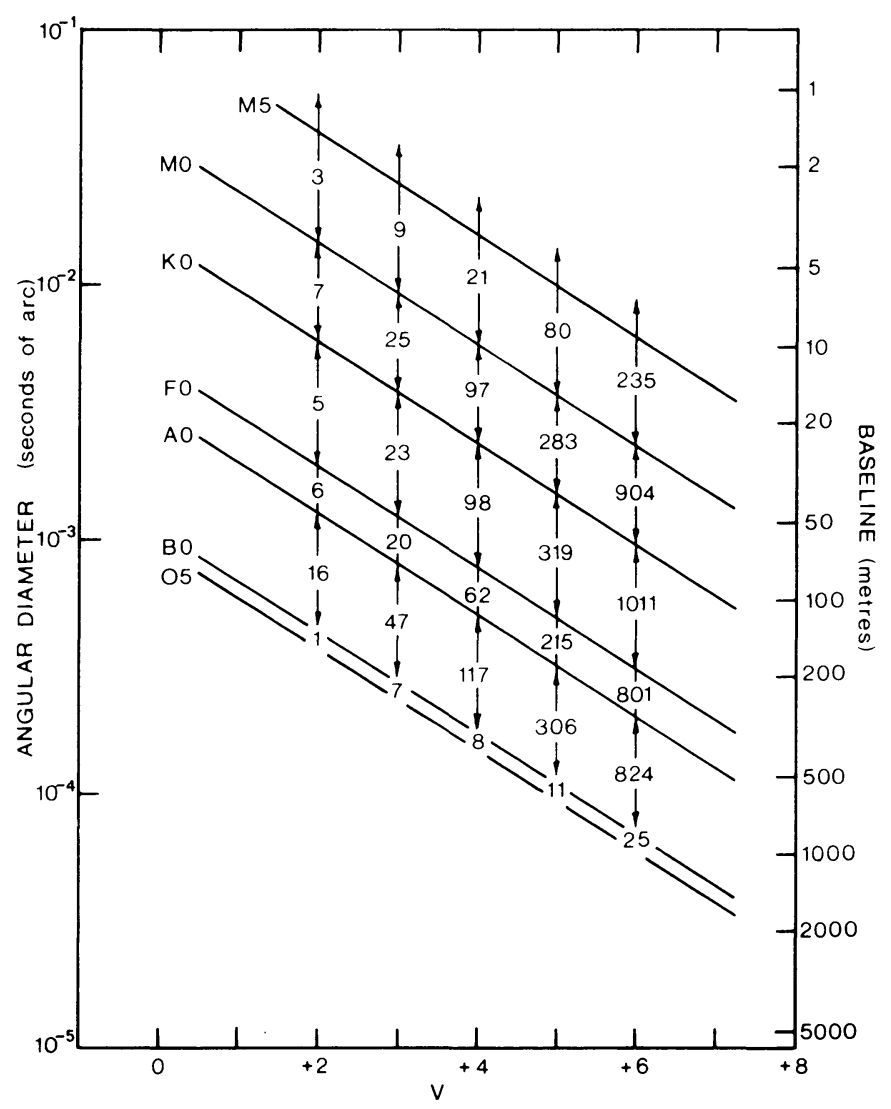

Fig. 2. Distribution of stars with respect to apparent brightness and angular size. The diagonal lines represent mean relationships for the spectral types shown and the figures give the total number of stars in the Catalogue of Bright Stars (Hoffleit 1964) brighter than a given $V$ magnitude with declination $<+30^{\circ}$ for each spectral group (e.g. there are' 215 A-type stars with $\mathrm{V}<+5)$. The right-hand ordinate gives the interferometer baseline required for resolution $\left(|\gamma|^{2}=0.5\right)$ at a wavelength of $550 \mathrm{~nm}$. 
Figure 2 shows the distribution with respect to brightness and angular diameter of stars brighter than $\mathrm{V}=+6$ and with declination $<+30^{\circ}$. The figure suggests that angular diameters for a large sample of stars could be determined with an instrument capable of measuring stars to $\mathrm{V}=+4$. This is true for the middle range of spectral classes but a closer examination of the statistics reveals that for early 0 and for $M$ main-sequence stars greater sensitivity is required. To measure hot $O$ stars it is negessary to reach at least $V=+6$ and, in order to obtain a reascnable sample, $V($ limit) should exceed +7 . For mainsequence stars of spectral class MO and later it is also necessary to have $\mathrm{V}($ limit) $>+7$. In fact, the $\mathrm{V}($ limit) required to measure mainsequence stars increases towards later spectral classes and exceeds +9.5 at M5.

It can be seen from Figure 2 that an angular resolution of $\sim 2 \times 10^{-4}$ arcseconds would be sufficient for measuring all except the hottest stars but for these an angular resolution of $\sim 4 \times 10^{-5}$ arcseconds is required.

If a measured angular diameter is to be used to determine the emergent flux at the stellar surface $(F)$ and effective temperature $\left(T_{e}\right)$ then the aim should be to measure the angular diameter $(\theta)$ with an accuracy such that its contribution to the uncertainty in $F$ is no greater than that from the integrated absolute flux received from the star (f). F, f, $\theta$ and $T_{e}$ are related by

$$
F=4 f / \theta^{2}=\sigma T_{e}^{4}
$$

where $\sigma$ is the stefan-Boltzmann constant. From equation (1) it can be seen that the target is to make $2 \sigma(\theta) \lesssim \sigma(f)$ where $\sigma(\theta)$ and $\sigma(f)$ are the fractional uncertainties in $\theta$ and $f$.

Figure 3 is a plot of $2 \sigma(\theta)$ and $\sigma(f)$ against $(B-V)_{\circ}$ for the 32 stars measured with the intensity interferometer. The data is taken from Code et al. (1976). For $(B-V)_{0} \leqslant-0.15$ the uncertainties are generally well matched largely because of the increasing uncertainty in the integrated fluxes for the hotter stars. However, for $(B-V) \circ \geqslant-0.15$, the uncertainty in the integrated flux is of the order of $\pm 4 \%$ whereas $2 \sigma(\theta)$ ranges from $\sim \pm 4 \%$ up to $\sim \pm 28 \%$. On the basis of the evidence shown in Figure 3 future angular diameter measurements should aim at reducing $\sigma(\Theta)$ to $\lesssim \pm 2 \%$.

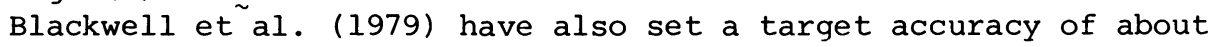
$2 \%$ in $\theta$ and $1 \%$ in $\mathrm{T}_{e}$ for their infrared flux method which would be commensurate with that of the oscillator strengths being measured at Oxford.

The targets to be aimed at in future angular diameter determinations thus include $\mathrm{V}$ (Iimit) $>+7$ for spectral classes earlier than MO increasing to $\mathrm{V}$ (limit) $>+9.5$ for M5 main-sequence stars, an angular resolution of $2 \times 10^{-4}$ arcseconds decreasing to $4 \times 10^{-5}$ arcseconds for a sample of hot $O$ stars, and generally an accuracy of $\leqslant \pm 2 \%$.

The difficulties involved in reaching these targets are formidable and have led Barnes and Evans (Barnes \& Evans 1976; Barnes et al. 1976; Barnes et al. 1978) to develop an empirical relationship between visual surface brightness and color index based on existing angular diameter 


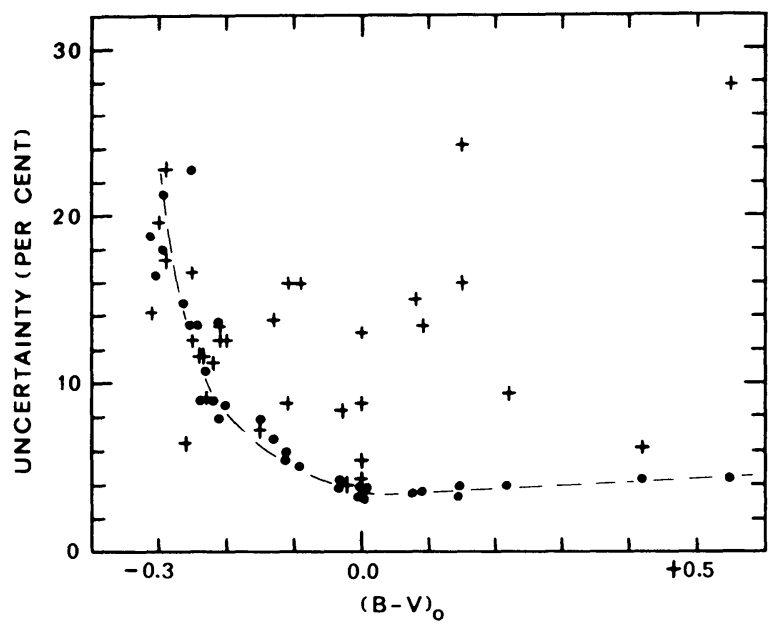

Fig. 3. The uncertainty in integrated flux received from a star and twice the uncertainty in angular diameter (t) as a function of $(B-V)_{0}$ for the 32 stars measured with the intensity interferometer. The broken line indicates the trend of the uncertainties in the fluxes.

measurements. While it is not fundamental in the sense that it is calibrated with empirical data it has nevertheless found numerous applications (Barnes et al. 1978) in the absence of direct angular diameter measurements. An important feature of the relationship is the fact that it can be applied to stars which are below the current sensitivity of techniques for direct angular diameter determinations. Its usefulness would be extended by improvements in calibration which would be brought about by angular diameter determinations of improved accuracy. This is particularly true in the range of spectral classes $A$ to $G$ where the visual surface brightness relationship is least well defined.

The infrared flux method has been devised by Blackwell \& Shallis (Blackwell \& Shallis 1977; Blackwell et al. 1978; Blackwell et al. 1979) with the stated intention of providing a fundamental techinique that will give angular diameters and effective temperatures for selected stars of virtually any spectral type to a magnitude limit of at least +7 or +8 with an accuracy at least equal to that attainable by other methods and which is completely independent of results from other methods. They have shown (Blackwell et al. 1979) that their results are insensitive to the model atmosphere used, at least for $\mathrm{T}_{\mathrm{e}} \lesssim 8000 \mathrm{~K}$, and that the interstellar extinction is not a siqnificant problem. So far the method has given angular diameters that are larger by $3.8 \%$ on average than those measured with the intensity interferometer. This discrepancy may be the result of errors arising from the absolute infrared flux calibration which is crucial to the success of the infrared flux method. The cause of the discrepancy needs to be resolved and Blackwell and his colleagues are endeavoring to improve the infrared 
flux calibration. It is also important in this context to improve the spectral coverage and accuracy of direct angular diameter measurements to provide further crosschecks on the results.

The targets for future angular diameter measurements have been discussed in the context of surface fluxes and effective temperatures. Stellar radii, and hence luminosities, can also be determined from angular diameter measurements for stars of known parallax. The usefulness of this application is currently limited by the accuracy of existing trigonometric parallaxes. The radii of stars of known trigonometric parallax can only be obtained with an accuracy better than $\pm 20 \%$ for spectral classes $A O$ and later (Davis 1979a). From one site there are some 100 stars brighter than $\mathrm{V}=+10$ with trigonometric parallaxes known to better than \pm 20 \% but only $\sim 12$ better than $\pm 5 \%$. Thus the targets set above are more than adequate to match existing parallaxes and, in fact, will be adequate in almost all cases for radii determined from parallaxes determined with the astrometric satellite HIPPARCOS (mean error 0.002 arcseconds (H $\varnothing \mathrm{g}$ 1978)). This conclusion is also true for the determination of masses of single stars by the method proposed by Blackwell et al. (1978) since it too involves parallaxes.

High angular resolution observations of suitable double-lined spectroscopic binaries will also enable stellar masses and radii to be determined. The case of binary stars is discussed by McAlister (1985), but the targets discussed here are appropriate for this application (Davis 1983).

\section{THE DIRECT MEASUREMENT OF ANGULAR DIAMETERS}

Of the techniques available for the direct measurement of stellar angular diameters only long baseline interferometry has the potential to meet all the targets set in the previous section although baselines in excess of $1 \mathrm{~km}$ will be required to achieve an angular resolution of $4 \times 10^{-5}$ arcseconds at optical wavelengths as can be seen from Figure 2 .

The limitation in angular resolution of the lunar occultation technique restricts it to the measurement of luminous cool stars and it is here that it has made a major contribution as shown by Figure 1. Although the technique is capable of measuring angular diameters down to the 1-2 milliseconds of arc range (Ridgway 1979) the accuracy deteriorates below $\sim 3$ milliseconds of arc and, with only a few notable exceptions, accurate angular diameter measurements will remain restricted to luminous stars of late $G, K$ and $M$ spectral classes.

The angular resolution of amplitude and speckle interferometry using a single telescope is restricted to $\sim 15$ milliseconds of arc by the diameters of existing telescope apertures. Although the angular diameters of 9 stars have been determined by each technique with an accuracy better than $\pm 20 \%$, in the cases of 4 of the speckle results and 7 of the amplitude results the angular diameters have been determined with greater accuracy by other methods. This is shown by the surviving results appearing in Figure 1. Even with the next generation telescopes 
under consideration these techniques will not make a significant contribution to stellar angular diameter determinations.

There are several possible approaches to long baseline interferometry but since intensity interferometry has been by far the most successful it would seem to be the obvious approach to pursue. A possible design for a very large intensity interferometer with increased sensitivity, resolution and accuracy has been discussed by Hanbury Brown (1974) and Davis (1975). It appears that the resolution and accuracy targets given in section 3 could be reached with an intensity interferometer but that an increase in sensitivity of $\sim 80$ times over the Narrabri instrument, which had a limiting blue magnitude $B$ (limit) $\sim+2.5$ (Hanbury Brown et al. 1974), is all that can be achieved without great difficulty and prohibitive cost. There is no doubt that a very large intensity interferometer could be built and that it would work but a modern amplitude interferometer offers the possibility of greater sensitivity at lower cost. If reasonable parameters are assumed it can be shown that it would be necessary to use apertures of the order of $30 \mathrm{~m}$ in an intensity interferometer in order to achieve the same sensitivity as an amplitude interferometer with $10 \mathrm{~cm}$ diameter apertures.

The development of amplitude interferometers has been inhibited by two main areas of difficulty in the past. One is associated with the severe requirements of mechanical stability imposed by the wavelength of light and the other is the problem of making accurate measurements in the presence of the disruptive effects of atmospheric turbulence on the incoming wavefronts. The problems imposed by the atmosphere can be avoided by an interferometer in space (see for example Labeyrie 1978; Stachnik \& Labeyrie 1984) but since the space proposals are still at an early stage of development and are not likely to contribute measurements of angular diameters within at least the next decade they will not be discussed here. The use of lasers, and modern control, detection and data handling techniques offers the possibility of overcoming the mechanical and atmospheric problems in a ground-based amplitude interferometer and this, together with the sensitivity advantage of an amplitude interferometer, are the reasons why longbaseline optical interferometers currently under development are all amplitude instruments.

A large aperture amplitude interferometer is being developed at CERGA (Labeyrie 1978) and small aperture amplitude interferometers are being developed at the Universities of Sydney (Davis 1979b) and Maryland (Liewer 1979). In the CERGA interferometer the Coude images from two telescopes, whose apertures are large compared with Fried's atmospheric coherence diameter $r_{0}$ (Fried \& Mevers 1974), are relayed to a central station where fringes appear in the superposed images providing the optical paths are matched within the tolerances set by the coherence length of the light. The prototype instrument (I2T) using $25 \mathrm{~cm}$ diameter aperture telescopes has been successfully used out to baselines of $\sim 40 \mathrm{~m}$ to determine $|\gamma|$ for bright stars with

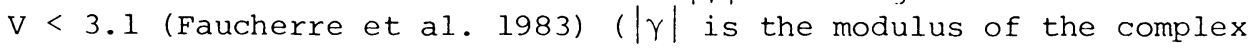
degree of coherence (Born \& Wolf 1964) equal to Michelson's fringe visibility). Work is in progress to develop telescopes with aperture diameters of $1.52 \mathrm{~m}$ (Labeyrie 1978) for use in this type of instrument. 
In the small aperture approach the apertures are restricted to $\lesssim r_{0}$ (which has a value of $\sim 10 \mathrm{~cm}$ at a good site) and the effects of atmospheric turbulence are then removed in real time from the measurement of $|\gamma|$. How this is done in the instrument being developed at the University of Sydney will be described in the following section. The relative merits of the large aperture and small aperture approaches have been outlined by Davis (1976). It is not yet clear which will prove, most suited to stellar angular diameter determinations although it is to be hoped that both will be successful so that stars may be observed in common to provide crosschecks. The large aperture approách is expected to have greater sensitivity but a small amplitude interferometer, because it incorporates removal of atmospheric effects in real time from the measurement of $|\gamma|$, should be capable of achieving greater accuracy.

\section{A SMALL APERTURE MODERN MICHELSON STELLAR INTERFEROMETER}

A small aperture modern Michelson stellar interferometer is under development at the University of Sydney and a prototype instrument is currently nearing completion in the grounds of the Australian National Measurement Laboratory at West Lindfield, near Sydney.

The prototype instrument represents a logical step following the work of Twiss and Tango with the Monteporzio $2 \mathrm{~m}$ amplitude interferometer (Tango 1979a). The principles and theory of a small aperture amplitude interferometer have been discussed by Tango and Twiss (Tango 1979b; Tango \& Twiss 1980) and they apply directly to the prototype instrument. Since the design considerations for the prototype (Davis 1979b) and, recently, a more detailed description of the design and layout (Davis 1984) have been published only a very brief summary of the key features will be given here.

The prototype interferometer has a fixed $11.4 \mathrm{~m}$ north-south baseline and coelostats mounted on plinths at each end of the baseline to steer starlight into a central laboratory. This laboratory houses the optical system of the interferometer which includes a path length compensator to equalise the light paths from the star to the beamsplitter where the beams from the two sides of the instrument are combined.

\subsection{The Elimination of Atmospheric Problems}

The effect of atmospheric turbulence is to produce time dependent deformations of the wavefronts reaching the coelostats which will reduce the observed visibility $|\gamma|$ below the true value. The atmospheric effects are circumvented by restricting the effective apertures of the coelostats to $\leqslant r_{0}$ (the maximum primary beam diameter is $10 \mathrm{~cm}$ ) so that 'flat' sections of wavefront are selected and also by reducing the mean tilts of the wavefronts using optical servo systems. These steps produce a significant reduction in the loss in $|\gamma|$ (Tango \& Twiss 1980) and the residual loss can be estimated from auxilliary measurements. For this reason an auxilliary interferometer is being developed to provide real time measurements of $r_{0}$ which will be used 
to select the effective aperture size and to provide a correction factor for the residual losses due to wavefront curvature. The loss arising from uncompensated wavefront tilt fluctuations and shot noise in the tilt correcting servo signals, which will cause the tilt correcting mirrors to dither randomly, can be estimated from the noise power of the servo error signal (Tango \& Twiss 1980). This procedure has been used with very good results in the Monteporzio interferometer (Tango 1979a).

The tilt corrected wavefronts from the two arms of the interferometer are arranged to interfere at nominally zero angle in a beamsplitter so that, in the absence of aberrations, the complementary output beams are uniformly illuminated. The irradiances of the beams are proportional to $(1+|\gamma| \cos \phi)$ and $(1-|\gamma| \cos \phi)$ where $\phi$ is a randomly varying phase angle resulting from atmospheric optical path length fluctuations and path equalisation errors. The sampling time $\tau$ of the photon counting detectors measuring the irradiance in the two beams is made short so that changes in $\phi$ are not significant during T which is expected to be in the range $1-10 \mathrm{~ms}$. The photocounts registered by the two detectors in $\tau$ are processed by a correlator which basically measures the square of the difference between the two signals. The data are integrated for a total observing time $T$ and analysed to yield $2|\gamma|^{2}\left\langle\cos ^{2} \phi\right\rangle$, where the brackets indicate an average over $T$. If the phase is a uniform random variable $2|\gamma|^{2}\langle\cos \phi\rangle$

becomes $|\gamma|^{2}$, the square of the fringe visibility. Provision has been made to introduce a path difference of $\lambda / 4$ between the two interferinc: beams halfway through the sampling period $\tau$ so that the correlator gives $\left.2|\gamma|^{2}<\cos ^{2} \phi+\sin ^{2} \phi\right\rangle$ instead of $\left.2|\gamma|^{2}<\cos ^{2} \phi\right\rangle$ so that it will not be necessary to assume that the phase is a uniform random variable to obtain $|\gamma|^{2}$.

Tango and Twiss (1980) have shown that irradiance fluctuatons due to scintillation will have a guite negligible effect on the measurement of $|\gamma|^{2}$ in a small aperture amplitude interferomter.

\subsection{The Elimination of Mechanical Problems}

In order to provide the required mechanıcal stability the component parts of the prototype interferometer are all mounted on massive reinforced concrete plinths anchored in a monolithic layer of sandstone approximately $1 \mathrm{~m}$ below ground level. Seismometer tests carried out prior to site selection established that ambient vibration levels were acceptable.

Particular care has been taken in the design and construction of the coelostat mounts to minimize vibration and path length changes with orientation. The only other component mechanically driven during an observation is a truck carrying a retroreflector in the optical path length compensator (OPLC) and this is the most critical component of the interferometer. This is not simply because it has to match the optical paths rrom the star to within a tolerance set by the optical bandwidth but because it also has to meet a particularly severe requirement regarding the smoothness of its motion. Not only has the reflector to move at the correct velocity but any error or irregularity in the 
velocity in the sampling time $\tau$ will result in a loss of coherence and an error in the measured $|\gamma|^{2}$. The tolerances for the OPLC to keep losses in $|\gamma|^{2} \leq 1 \%$ have been established by Tango and Twiss (1980) and the OPLC for the prototype interferometer, which has been designed and built to meet these tolerances, has been described by Davis (1984).

\subsection{The Prototype Program}

The prototype interferometer is nearing completion and it will be used to establish the accuracy and reliability that can be achieved in measurements of $|\gamma|^{2}$ through the atmosphere. In developing the design of the interferometer considerable effort has been made to keep

individual sources of error in the measurement of $|\gamma|^{2}$, both atmospheric and mechanical, to $< \pm 1 \%$ so that, at least for bright stars, an accuracy of $\leqslant \pm 2 \%$ in $|\gamma|^{2}$ can be achieved. An accuracy of \pm 0.02 in $|\gamma|^{2}$ measured at the optimum baseline $d_{0} \simeq 1.3 \times 10^{-4} \lambda / \theta$, where $\lambda$ is the wavelength of observation in $\mathrm{nm}$ and $\theta$ is the angular diameter in arcseconds, corresponds to an accuracy of $\sim 2.5 \%$ in the angular diameter. The limiting accuracy is ultimately expected to be set by the tilt-correcting servo and this will deteriorate for fainter stars. The faintest magnitude at which $\pm 2 \%$ accuracy can be achieved depends on how well the corrections for residual losses due to atmospheric effects can be established but at this stage it appears that V(limit) will be in the range +7 to +8 (Tango 1979b). The accuracy will deteriorate rapidly below $\mathrm{V}=+8$ to +9 .

The prototype program is the foundation for the major high angular resolution interferometer that we plan to build and the component parts of the prototype instrument have been designed to become the heart of the long baseline instrument. The preliminary specification includes baselines extending eventually to a kilomecer or more with the same limiting magnitude and accuracy as for the prototype interferometer. This would enable angular sizes down to $\sim 5 \times 10^{-5}$ arcseconds to be measured with an accuracy of the order of $\pm 2 \%$ to a $V($ limit) of +7 to +8 .

Apart from the increase in baseline lengths and the corresponding increase in the effective length of the OPLC there are two additional features which will be included in the major instrument. Firstly, for baselines in excess of $\sim 50 \mathrm{~m}$ it will be necessary to use an automatic fringe tracking system (Davis 1984) and a system such as that developed by Shao and Staelin (1980) will be incorporated. Secondly, the single spectral channel detectors used in the prototype instrument for the determination of $|\gamma|^{2}$ will be replaced by photon counting linear array detectors covering a range of the spectrum. This will enable simultaneous observations to be made in spectral lines as well as in multiple channels in the continuum.

A search for a suitable site with stable rock near the surface and large enough to accommodate either east-west and/or north-south baselines of at least $1 \mathrm{~km}$ in length is under way. 
6. CONCLUSIONS

The targets for future angular diameter determinations suggested in Section 3, namely $\mathrm{V}(\mathrm{limi})>+7$, angular resolution of $\sim 4 \times 10^{-5}$ arcseconds and an accuracy of $\leqslant \pm 2 \%$, appear to be within the reach of a ground based small aperture amplitude interferometer of the type being developed at the University of Sydney. They may also be within the reach of the large aperture approach being developed by Labeyrie and his colleagues at CERGA.

The importance of observing stars by more than one technique is stressed. In due course it will be desirable to establish a list of reference stars visible from both the Northern and Southern Hemispheres, including stars occulted by the Moon, for comparison and calibration purposes.

The prospects for contributions by high angular resolution measurements to the determination and calibration of fundamental stellar quantities during the next decade are excellent.

\section{ACKNOWLEDGEMENTS}

The University of Sydney stellar interferometer program is supported by the Australian Research Grants Scheme, the University of Sydney Research Grants Committee and the science Foundation for Physics within the University of Sydney.

\section{REFERENCES}

Barnes, T.G. \& Evans, D.S. 1976, Mon. Not. R. Astron. Soc., 174, 489. Barnes, T.G., Evans, D.S. \& Moffett, T.J. 1978, Mon. Not. R. Astron. Soc., 183, 285.

Barnes, T.G., Evans, D.S. \& Parsons, S.B. 1976, Mon. Not. R. Ascron. Soc., 174, 503 .

Beavers, W.I., Cadmus, R.R. \& Eitter, J.J. 1981, Astron. J., 86, 1404.

Blackwe1l, D.E., Petford, A.D. \& Shallis, M.J. 1978, in Colloquium on European Satellite Astronomy, Padua, 1978 June 5-7, p.223.

Blackwell, D.E. \& Shallis, M.J. 1977, Mon. Not. R. Astron. Soc., 180, 177.

Blackwell, D.E., Shallis, M.J. \& Selby, M.J. 1979, Mon. Not. R. Astron. Soc., 188, 847.

Blazit, A., Bonneau, D., Koechlin, L. \& Labeyrie, A. 1977, Astrophys. J., 214, L79.

Born, M. \& W̄olf, E. 1964, Principles of Optics, Oxford: Pergamon Press.

Code, A.D., Davis, J., Bless, R.C. \& Hanbury Brown, R. 1976, Astrophys. J., 203, 417.

Currie, D.G., Knapp, S.L., Liewer, K.M. \& Braunstein, R.H. 1976, University of Maryland Technical Report, \#76-125.

Davis, J. 1975, in Multicolor photometry and the Theoretical HR Diagram, ed. A.G.D. Philip \& D.S. Hayes, Dudley Observatory Report No.9,p.199. 
Davis, J. 1976, Proc. Astron. Soc. Australia, 3, 26. Davis, J. 1979a, in High Angular Resolution Stellar Interferometry (IAU Colloquium No. 50), ed. J. Davis \& W.J. Tango, p.l.1. Chatterton Astronomy Department, University of Sydney.

Davis, J. 1979b, in High Angular Resolution Stellar Interferometry (IAU Colloquium No. 50), ed. J. Davis \& W.J. Tango, p.14.1. Chatterton Astronomy Department, University of Sydney.

Davis, J. 1983, in Current Techniques in Double and Multiple star Research (IAU Colloquium No. 62), ed. R.S. Harrington \& O.G. Franz, p.191. Lowell Observatory Bulletin No. 167. Davis, J. 1984, in Indirect Imaging, ed. J.A. Roberts, p.125. Cambridge University Press.

Di Benedetto, G.P. \& Conti, G. 1983, Astrophys. J., 268, 309. Dunham, D.W., Evans, D.S. \& Vogt, S.S. 1975, Astron. J., 80, 45. Evans, D.S. 1957, Astron. J., 62, 83.

Faucherre, M., Bonneau, D., Koechlin, L. \& Vakili, F. 1983, Astron. Astrophys. , 120, 263.

Fried, D.L. \& Mevers, G.E. 1974, Applied Optics, 13, 2620 (Correction in Applied Optics, 14, 2567).

Gezari, D.Y., Labeyrie, A. \& Stachnik, R.V. 1972, Astrophys. J., $173, \mathrm{Ll}$.

Hanbury Brown, R. 1974, The Intensity Interferometer. London: Taylor \& Francis.

Hanbury Brown, R. 1985, in Calibration of Fundamental Stellar Quantities (IAU Symposium No. 11l), ed. D. S. Hayes, L. E. Pasinetti and A. G. D. Philip, (Reidel, Dordrecht), p. 185.

Hanbury Brown, R., Davis, J. \& Allen, L.R. 1974, Mon. Not. R. Astron. Soc., 167, 121 .

Herbison-Evans, D., Hanbury Brown, R., Davis, J. \& Allen, L.R. 1971, Mon. Not. R. astron. Soc., 151, 161.

Hoffleit, D. 1964, Catalogue of Bright Stars, Yale University Observatory.

$\mathrm{H} \phi \mathrm{g}, \mathrm{E} . \mathrm{1978}$, in Colloquium on European Satellite Astronomy, Padua, 1978 June $5-7$, p.7.

Labeyrie, A. 1978, Ann. Rev. Astron. Astrophys., 16, 77.

Liewer, K.M. 1978, in High Angular Resolution Stellar Interferometry (IAU Colloquium No. 50), ed. J. Davis \& W.J. Tango, p.8.l. Chatterton Astronomy Department, University of Sydney.

McAlister, H. A. 1985, in Calibration of Fundamental Stellar Quantities (IAU Symposium No. Ill), ed. D. S. Hayes, L. E. Pasinetti and A. G. D. Philip, (Reidel, Dordrecht), p. 97.

Nelson, M.R. 1975, Astrophys. J., 198, 127. Radick, R.R. 1981, Astron. J., 86, 1685.

Ridgway, S.T. 1979, in High Angular Resolution Stellar Interferometry (IAU Colloquium No. 50), ed. J. Davis \& W.J. Tango, p.6-1. Chatterton Astronomy Department, University of Sydney.

Ridgway, S.T., Jacoby, G.H., Joyce, R.R., Siegel, M.J. \& Wells, D.C. 1982, Astron. J., 87, 1044.

Ridgway, S.T., Jacoby, G.H., Joyce, R.R. \& Wells, D.C. 1980, Astron. J. , 85, 1496 .

Ridgway, S.T., Wells, D.C. \& Carbon, D.F. 1974, Astron. J., 79, 1079. 
Shao, M. \& Staelin, D.H. 1980, Applied Optics, 19, 1519.

Stachnik, R.V. \& Labeyrie, A. 1984, Sky \& Telescope, 67, 205.

Tango, W.J. 1979a, in High Angular Resolution Stellar Interferometry (IAU Colloquium No. 50), ed. J. Davis \& W.J. Tango, p.13.1.

Chatterton Astronomy Department, University of Sydney.

Tango, W.J. 1979b, in High Angular Resolution Stellar Interferometry (IAU Colloquium No. 50), ed. J. Davis \& W.J. Tango, p.12.1. Chatterton Astronomy Department, University of Sydney.

Tango, W.J. \& Twiss, R.Q. 1980, in Progress in Optics XVII, ed.

E. Wolf, p.239. Amsterdam: North Holland.

Welter, G.L. \& Worden, S.P. 1980, Astrophys. J., 242, 673.

White, N.M. 1974, Astron. J., 79, 1076 .

White, N.M. 1980, Astrophys. J., 242, 646.

Worden, S.P. 1975, Astrophys. J., 201, L69.

Worden, S.P. 1976, Pub. Astron. Soc. Pacific, 88, 69.

Liscussion of this paper occurred after the paper by McAlister. 